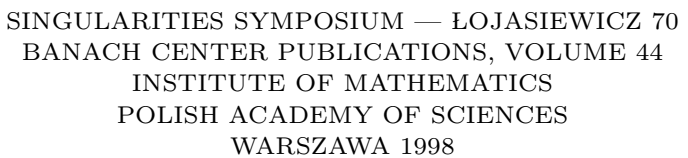

\title{
ON SYMMETRIC SEMIALGEBRAIC SETS AND ORBIT SPACES
}

\author{
LUDWIG BRÖCKER \\ Mathematisches Institut, Universität Münster \\ Einsteinstraße 62, D-48149 Münster, Germany \\ E-mail:Broe@math.uni-muenster.de
}

\begin{abstract}
For a symmetric (= invariant under the action of a compact Lie group $G$ ) semialgebraic basic set $C$, described by $s$ polynomial inequalities, we show, that $C$ can also be written by $s+1 G$-invariant polynomials. We also describe orbit spaces for the action of $G$ by a number of inequalities only depending on the structure of $G$.
\end{abstract}

Introduction. Let $Y$ be a real algebraic affine $G$-variety where $Y$ is defined over $\mathbb{R}$ and $G$ is a compact Lie group acting on $Y$ (or $\mathbb{R}[Y]$ respectively). In all this work we only consider real points of real varieties. So we just write $Y$ for which should be called $Y(\mathbb{R})$. There are some natural questions:

- What is the structure of the orbit space $X$ of $Y$ under the action of $G$ ?

- If $C \subset Y$ is semialgebraic and $G$-invariant, can $C$ be described by $G$-invariant elements?

- If $C \subset Y$ is semialgebraic, $G$-invariant and basic open (or closed), can $C$ be described by $G$-invariant elements as a basic open (or closed) set, and how many inequalities are needed?

The first question was answered by Procesi and Schwarz [Pro-Schw]: Let $Y / / G:=$ $\operatorname{Spec}\left(\mathbb{R}[Y]^{G}\right)$ and $Z=(Y / / G)(\mathbb{R})$. Then $X=p(Y) \subset Z$ where $p: Y \rightarrow Z$ is induced by the inclusion $\mathbb{R}[Y]^{G} \rightarrow \mathbb{R}[Y]$ and $X$ is a basic closed subset of $Z$ which is defined by saying that a certain symmetric matrix over $\mathbb{R}[Y]^{G}$ is positive semidefinite (see also Section 5). However, this description of $X$ leads to a lot of inequalities, depending on the number of generators for $\mathbb{R}[Y]^{G}$. We will show, that the number of inequalities for a description of $X$, at least generically, that means, up to a set of smaller dimension, depends only on the structure of $G$.

As to the second question, the answer can easily seen to be positive.

1991 Mathematics Subject Classification: 14G30, 14L30.

Received by the editors: December 27, 1996; in the revised form: November 6, 1997.

The paper is in final form and no version of it will be published elsewhere. 
Concerning the third question, the basic open sets are easier to treat. If $C \subset Y$ is $G$-invariant and basic open, described by $s$ inequalities, then $C$ can also be described by at most $s+1 G$-invariant inequalities or even by $s G$-invariant inequalities in case that $G$ is finite. It is an open problem, whether this better estimate holds in general.

For basic closed sets, again we only can show that $S$ can be described as a basic closed set by invariant inequalities and generically one needs $s+1$ of them (or $s$ if $G$ is finite).

For finite groups $G$ the above questions and answers can be generalized essentially. So let $G$ act on $A$ where $A$ is a arbitrary commutative ring with unit and let $B:=A^{G}$. Let us denote by $\operatorname{Sper}(A)$ the real spectrum of $A$ and correspondingly for $B$. Here I hope that the reader is a little bit familiar with real spectra (I refer to [B-C-R, Chap. 7], [Kn-Schei], [Be2] and [B]). Now, concerning the above problems we just replace $Y$ by $\operatorname{Sper}(A), Z$ by $\operatorname{Sper}(B)$ and $X$ by $\tilde{X}:=p(\operatorname{Sper}(A))$ where $p: \operatorname{Sper}(A) \rightarrow \operatorname{Sper}(B)$ is induced by the inclusion $B \hookrightarrow A$. In case that $\operatorname{Sper}(A)$ is noetherian we get corresponding results.

The most elementary situation appears, when $A / B$ is a Galois extension of fields with Galois group $G$. Here $\tilde{X}$ can be described by $k$ inequalities where $k$ is the maximal number of mutually commuting independent involutions in $G$. This result is due to Scheiderer [Schei2]. We present the proof in Section 3, since the methods which are used for this have to be introduced anyway. In fact the key ingredient is the theory of fans which, for convenience of the reader, is briefly explained in Section 2 (for more details see [A-B-R, Chap. 2-6], [B] and [MarI]). After studying extensions $A / B, B=A^{G}$ for finite groups $G$ in Section 4, in Section 5 we turn to $\mathbb{R}$-varieties $Y$ and the action of compact Lie groups $G$ on $Y$. If $G$ is finite, the "tilde" correspondence, which relates semialgebraic sets to constructible sets in real spectra, immediately leads to the desired results. For infinite $G$, we use étale slices in the sense of Luna $[\mathrm{Lu}]$ in order to get a reduction to finite $G$. But this works only locally, so that for our estimates we need one more inequality than for finite $G$.

1. The action of a finite group on real spectra. Let $A$ be a commutative ring with unit. By $\operatorname{Sper}(A)$ we denote the real spectrum of $A$. For information about $\operatorname{Sper}(A)$ see [B-C-R], [Kn-Sche], [Ma], [B], [Be2], [A-B-R]. For $a \in A$ we write $\{a>0\}$ instead of $\{\alpha \in \operatorname{Sper}(A) \mid \alpha(a)>0\}$. Similarly, we use $\{a=0\},\{a \geq 0\}$ and conjunctions, for instance, we write $\{a>0, b>0\}$ instead of $\{a>0\} \cap\{b>0\}$.

We shall always assume that $\operatorname{Sper}(A)$ is noetherian with respect to the Zariski topology. From this we get the following obvious

Proposition 1.1. Let $C \subset \operatorname{Sper}(A)$ such that $C \cap \operatorname{Sper}\left(k_{\wp}\right)$ is constructible in $\operatorname{Sper}\left(k_{\wp}\right)$ for all (real) prime-ideals $\wp$ of $A$. Then $C$ is constructible in $\operatorname{Sper}(A)$.

Here, of course, $\operatorname{Sper}\left(k_{\wp}\right)$ is identified with $\{\alpha \in \operatorname{Sper}(A) \mid \operatorname{supp}(\alpha)=\wp\}$.

Now let $G$ be a finite group of automorphisms of $A$. We denote by $B=A^{G}$ the ring of invariant elements. Then $G$ also acts on $\operatorname{Sper}(A)$ via

$$
g(\alpha)(a)=\alpha\left(g^{-1}(a)\right)
$$

for $\alpha \in \operatorname{Sper}(A)$ and $a \in A$ and $g \in G$. In general, $A \supset B$ will not be a Galois extension in the sense of [DM-I, Chap. III]. For $a \in A$ we denote by $\operatorname{tr}(a)$ the trace $\sum_{g \in G} g(a)$. Let $p: \operatorname{Sper}(A) \rightarrow \operatorname{Sper}(B)$ be the projection, induced by the inclusion $i: B \rightarrow A$.

Proposition 1.2. $\tilde{X}:=p(\operatorname{Sper}(A))=\bigcap_{a \in A}\left\{\operatorname{tr}\left(a^{2}\right) \geq 0\right\}$. 
Proof. Clearly $\tilde{X}$ is contained in the right-hand side. Conversely, let $\beta \in \operatorname{Sper}(B)$ such that $\beta$ cannot be extended to $A$. Consider $\beta \subset B$ as positive cone and let

$$
T:=\left\{\sum a_{i}^{2} b_{i} \mid a_{i} \in A, b_{i} \in \beta\right\} .
$$

Then $T \subset A$ is a precone, if $-1 \notin T$. By a basic result of Prestel [Pr, $\S 1]$ such a precone can be extended to some $\alpha \in \operatorname{Sper}(A)$ which extends $\beta$. So we must have

$$
-1=\sum_{i=1}^{m} a_{i}^{2} b_{i}
$$

for suitable $m \in \mathbb{N}, a_{i} \in A$ and $b_{i} \in \beta$. This leads to

$$
\sum_{i=1}^{m} \operatorname{Tr} a_{i}^{2} b_{i}=-g
$$

where $g=\#(G)$. Therefore $\beta\left(\operatorname{Tr}\left(a_{i}^{2}\right)\right)<0$ for at least one $i$.

Remark 1.3. By Proposition 1.2 the space $\tilde{X}$ is a saturated subset of $\operatorname{Sper}(B)$ in the sense of Marshall [Mar] or just a subspace in the frame of spaces of signs in the sense of [A-B-R, Chap. III]. That means, that $\tilde{X}$ shares the essential properties with real spectra of rings. Note that $\tilde{X}$ is noetherian. Hence a result like Proposition 1.1 holds also for subsets $C \subset \tilde{X}$. Moreover, if $\tilde{X} \cap \operatorname{Sper}(k(p))$ is constructible for all real prime ideals of $B$, the $\tilde{X}$ is constructible. In Section 4 we will show that $\tilde{X}$ is in fact basic closed and we will gain an estimate for the minimal number of inequalities for $\tilde{X}$.

Proposition 1.4. Let $C \subset \operatorname{Sper}(A)$ be basic closed and $G$-invariant. Then

$$
C=\bigcap_{C \subset\{a \geq 0\}}\{\operatorname{tr}(a) \geq 0\} .
$$

Pr o of. Clearly, $C$ is contained in the right-hand side. Conversely, assume that $\alpha$ is contained in the right-hand side but $\alpha \notin C$. Let $\beta=p(\alpha)$. There exists some $a \in A$ with $C \subset\{a \geq 0\}$ and $\alpha(a)<0$, hence $\beta(\operatorname{tr}(a))<0$. Let

$$
T:=\left\{\sum b_{i} a_{i} \mid b_{i} \in \beta, a_{i} \in A \text { with } C \subset\left\{a_{i} \geq 0\right\}\right\} .
$$

If $-1 \notin T$, then $T$ is a precone which can be extended to $\tau \in \operatorname{Sper}(A)$ and $\tau$ extends $\beta$. One has $\tau(a) \geq 0$ and also $\tau(g(a)) \geq 0$ for $g \in G$ since $C$ is $G$-invariant, hence $\tau(\operatorname{tr} a)=$ $\beta(\operatorname{tr}(a)) \geq 0$. Contradiction.

So $-1=\sum b_{i} a_{i}$ for suitable $b_{i} \in \beta$ and $a_{i} \in A$ with $C \subset\left\{a_{i} \geq 0\right\}$, hence $-g=\sum b_{i} \operatorname{tr}\left(a_{i}\right)$. Thus $\beta\left(\operatorname{tr}\left(a_{i}\right)\right)<0$ for at least one $a_{i}$. This is a contradiction to the assumption.

Proposition 1.5. For all subvarieties $V \subset \tilde{X}$ the group $G$ acts transitively on the components of $p^{-1}(V)$.

P r o of. Suppose $p^{-1}(V)$ decomposes into $G$-invariant Zariski-closed subsets $W_{1}, W_{2}$. We find $a_{i} \in A$ with $a_{i} \neq 0$ on $W_{i}$ and $a_{i}=0$ on $W_{j}, j \neq i$ for $i=1,2$. Then $\operatorname{tr}\left(a_{i}^{2}\right)$ does not vanish on $V$ but $\operatorname{tr}\left(a_{1}^{2}\right) \cdot \operatorname{tr}\left(a_{2}^{2}\right)$ does so. Contradiction. 
2. Fans. We keep the situation and notations of the previous section. We saw that the space $\tilde{X}=p(\operatorname{Sper}(A))$ is saturated and also, if $C \subset \operatorname{Sper}(A)$ is $G$-invariant and basic closed, then it can be described as an intersection by $G$-invariant functions. In fact, it will turn out that $p(\operatorname{Sper}(A))$ is basic closed and $C$ can be described as a finite intersection by $G$-invariant functions. So we want to know how many functions are needed. For this we need the notion of a fan.

Let $F \subset \operatorname{Sper}(A)$ be a subspace. That means, $F$ is of the form

$$
F=\bigcap_{d \in D}\{d>0\} \cap \bigcap_{e \in E}\{e \geq 0\} \quad \text { for } D, E \subset A .
$$

Then obviously any $a \in A$ defines a map

$$
\hat{a}: F \rightarrow \mathbb{F}_{3}
$$

by $\hat{a}(\sigma)=1$ if $\sigma(a)>0, \hat{a}(\sigma)=-1$ if $\sigma(a)<0$ and $\hat{a}(\sigma)=0$ if $\sigma(a)=0$.

Let $H$ be the set of all these functions and let $H^{*}=H \backslash 0$. Now $F$ is called a fan, if $H^{*}$ is a group, that is, all $\hat{a} \in H^{*}$ have values in $\{1,-1\}$, and if

$$
F=\left\{\sigma \in \hat{H}^{*} \mid \sigma(-1)=-1\right\} .
$$

Here $\hat{H}^{*}$ is the dual group of $H^{*}$ and $\sigma \in F$ can be considered as character on $H^{*}$ via $\sigma(\hat{a}):=\hat{a}(\sigma)$. If $F$ is finite, then clearly $\#(F)$ is a power of 2 .

Proposition 2.1 (Knebusch). Let $F \subset \operatorname{Sper}(A)$ be a fan with \# $(F) \geq 4$. Then all $\sigma \in F$ have the same support.

Proof. [A-B-R, III, Remark 3.13]

By this result, the investigation of fans reduces to the case where $A=K$ is a field. Let us fix some notations. Let $K$ be a field. For a valuation $v$ of $K$ we denote by $R_{v}$, $\bar{K}_{v}$ and $\Gamma_{v}$ the valuation ring, the residue field and the group of values respectively. We write $\Gamma_{v}$ additively and put $v(0)=\infty$. Recall that $\sigma \in \operatorname{Sper}(K)$ is called compatible with $v$ if $R_{v}$ is convex with respect to $\sigma$. Then $\sigma$ induces an ordering $\bar{\sigma} \in \operatorname{Sper}\left(\bar{K}_{v}\right)$.

Proposition 2.2 (trivialization of fans). Let $F \subset \operatorname{Sper}(K)$ be a fan and let $v$ be the finest valuation which is compatible with all $\sigma \in F$. Then $\#(\bar{F}) \leq 2$ where

$$
\bar{F}=\{\bar{\sigma} \mid \sigma \in F\} \subset \operatorname{Sper}\left(\bar{K}_{v}\right) .
$$

Proof. [A-B-R, VI, Theorem 1.6]

We say that the above valuation $v$ is associated to $F$. The significance of fans is due to the following most important result (see [A-B-R, V, Theorem 1.4]).

Theorem 2.3 (global generation formula). Let $C \subset \operatorname{Sper}(A)$ be constructible such that $C \cap \mathrm{Cl}_{Z}(\operatorname{Bd}(C))=\emptyset$. If for all finite fans $F \subset \operatorname{Sper}(A)$ one has

$$
\#(C \cap F) \text { divides \# }(F)
$$

and

$$
\text { \# }(F) \text { divides } 2^{k} \#(C \cap F) \text {, }
$$

then there are $g_{1}, \ldots, g_{k} \in A$ such that $C=\left\{g_{1}>0, \ldots, g_{k}>0\right\}$.

Here $\mathrm{Cl}_{Z}(\mathrm{Bd}(C))$ is the Zariski-closure of the boundary of $C$.

If one knows already that $C$ is basic open, but one wants information about the number of generators, only the second condition is needed. 
3. Extensions of fields. We return to the situation of Section 1 . So $G$ is a finite group which acts on the ring $A$ and $B=A^{G}$ is the ring of invariants. Now we assume that $B=K$ is a field and $A=L$ is a semisimple $K$-algebra. Then the situation is as follows: $L$ is a direct sum of mutually isomorphic fields, $L=L_{1} \oplus \ldots \oplus L_{r}$ and $G$ acts transitively on $\left\{L_{i}, i=1, \ldots, r\right\}$. Let $G_{i}$ be the stabilizer of $L_{i}$. Then $L_{i}^{G_{i}} \simeq K$, thus $L_{i} \supset K$ is a Galois extension with Galois group $G_{i} / C_{i}$ where

$$
C_{i}=\left\{g \in G_{i} \mid g(a)=a \quad \forall a \in L_{i}\right\} .
$$

In general, $C_{i}$ is not the trivial group. Let $m:=\left[L_{i}: K\right]$. Then $\operatorname{dim}(L)=m \cdot r$ and $\#(G)=m \cdot r \cdot \#\left(C_{i}\right)$.

Now let $\alpha \in \operatorname{Sper}(K)$ and assume that $\alpha$ admits at least one extension $\tilde{\alpha} \in \operatorname{Sper}(L)$. Then the total number of extensions is $m \cdot r$ and $G$ acts transitively on these. Similarly, for a valuation $v$ of $K$ by an extension $\tilde{v}$ of $v$ to $L$ we mean an extension $\tilde{v}$ in some $L_{i}$. Again, the total number of them is $d \cdot r$ and $G$ acts transitively on them. So let $\tilde{v}_{i j}$ be a valuation of $L_{i}$ which extends $v$. We denote by $D_{i j} \subset G_{i}$ the corresponding decomposition group, by $I_{i j}$ the inertia group and by $R_{i j}$ the ramification group. We will only consider the case where char $\left(\bar{K}_{v}\right)=0$, so $R_{i j}$ is trivial. Then

$$
I_{i j} \simeq\left(\Gamma_{\tilde{v}_{i j}} / \Gamma_{v}\right)^{\text {dual }} \text {. }
$$

Also $D_{i j} / I_{i j}$ is isomorphic to the Galois group of $\bar{K}_{\tilde{v}_{i j}} / \bar{K}_{v}$. As usual, set $e=\#\left(I_{i j}\right)$ and $f=\#\left(D_{i j} / I_{i j}\right)$. Note that $d=\left(G_{i}: D_{i j}\right)$. For the proposition below see also [Schei2, Section 1], [Be1, §4 Theorem 2.1].

Proposition 3.1. Let $\alpha \in \operatorname{Sper}(K)$ be compatible with the valuation $v$ of $K$. Assume that $\alpha$ admits at least one extension $\tilde{\alpha} \in \operatorname{Sper}(L)$ and let $\tilde{v}$ be an extension of $v$ in some $L_{i}$. Moreover, let $\bar{\beta} \in \operatorname{Sper}\left(\bar{K}_{\tilde{v}}\right)$ be any extension of $\bar{\alpha} \in \operatorname{Sper}\left(\bar{K}_{v}\right)$. Then

a) $I=I_{i j}$ is an elementary abelian 2-group.

b) There are exactly e extensions $\beta \in \operatorname{Sper}\left(L_{i}\right)$ of $\alpha$ which are compatible with $\tilde{v}$ and induce $\bar{\beta}$ on $\bar{K}_{\tilde{v}}$.

Pr o of. Assume, we have already a $\beta \in \operatorname{Sper}\left(L_{i}\right)$ with the properties in b). Let $\beta_{1}$ be another one. Then $\beta_{1}$ defines a character $\chi\left(\beta_{1}\right)$ on $\Gamma_{\tilde{v}} / 2 \Gamma_{\tilde{v}}$ :

$$
\chi\left(\beta_{1}\right)\left(\tilde{v}(b)+2 \Gamma_{\tilde{v}}\right):=\operatorname{sign}\left(\beta_{1}(b)\right) \operatorname{sign}(\beta(b)),
$$

and, by the Bear-Krull theorem [A-B-R, Prop. II, 3.3], $\chi$ defines a bijection between the set $B$ of all $\beta_{1}$ which are compatible with $\tilde{v}$ and induce $\bar{\beta}$ on $\bar{K}_{\tilde{v}}$ and the dual group of $\Gamma_{\tilde{v}} / 2 \Gamma_{\tilde{v}}$. Here we are only interested in those $\beta_{1}$ which extend $\beta$, so

$$
\chi\left(\beta_{1}\right) \mid \Gamma_{v} / 2 \Gamma_{v}=\mathrm{id} .
$$

Clearly, these $\chi\left(\beta_{1}\right)$ with $\beta_{1} \in B^{\prime}:=\left\{\beta_{1} \in B \mid \beta_{1}\right.$ extends $\left.\alpha\right\}$ form a 2-elementary subgroup $H$ of $\left(\Gamma_{\tilde{v}} / \Gamma_{v}\right)^{\text {dual }}=I$.

Now we have $m \cdot r$ extensions $\tilde{\alpha}$ of $\alpha$ in $\operatorname{Sper}(L)$. Each one is compatible with an extension $\tilde{v}$ of $v$. The number of the extensions $\tilde{v}$ is $r \cdot d$. The number of possible extensions of $\bar{\alpha}$ to $\bar{K}_{\tilde{v}}$ is $f$. So by what we have seen we get

$$
m r \leq d r f \cdot \#(H) \leq d r f \cdot \#(I)=m r,
$$

hence $H=I$ from which a) and b) follow.

The proposition below is due to Scheiderer [Schei2, Theorem 5.2]. For sake of completeness we give the proof here. 
Proposition 3.2. Let $\tilde{X}:=\{\alpha \in \operatorname{Sper}(K) \mid \alpha$ extends to $L\}$. Then $\tilde{X}$ is basic of the form $X=\left\{b_{1} \geq 0, \ldots, b_{k} \geq 0\right\}$, where $2^{k}$ is the order of an elementary abelian subgroup of $G_{i} / C_{i}$.

Proof. As a subset of $\operatorname{Sper}(K), \tilde{X}$ is constructible. So by Proposition 1.2 and an easy compactness argument $\tilde{X}$ is basic. If \# $(G)$ is odd, then $\tilde{X}=\operatorname{Sper}(K)$. So let \# $(G)$ be even and consider a finite fan $F \subset \operatorname{Sper}(K)$. According to Theorem 2.3 we have to compute the number

$$
2^{k}=\#(F): \#(\tilde{X} \cap F) .
$$

So let $\alpha \in X \cap F$ and let $v$ be the valuation associated to $F$ according to Proposition 2.2. Then there are $\bar{\alpha}, \bar{\beta} \in \operatorname{Sper}\left(\bar{K}_{v}\right)$ such that all $\beta \in F$ induce one of $\bar{\alpha}$ or $\bar{\beta}$ on $\bar{K}_{v}$.

Case 1. $F$ is a principal fan, that means, all $\beta \in F$ induce one and the same $\bar{\alpha}$ on $\bar{K}_{v}$.

Let $\tilde{\alpha} \in \operatorname{Sper}(L)$ be any extension of $\alpha$. Then, say, $\tilde{\alpha} \in \operatorname{Sper}\left(L_{1}\right), \tilde{\alpha}$ is compatible with a valuation $\tilde{v}$ of $L_{1}$, which extends $v$ and $\tilde{\alpha}$ induces $\bar{\gamma}$ on $\bar{K}_{\tilde{v}}$. By Proposition 3.1 any other $\beta \in \tilde{X} \cap F$ can be extended to some $\tilde{\beta} \in \operatorname{Sper}\left(L_{1}\right)$, compatible with $\tilde{v}$ such that $\tilde{\beta}$ induces $\bar{\gamma}$ on $\bar{K}_{\tilde{v}}$. But these $\tilde{\beta}$ are obtained by the Baer-Krull theorem: Firstly, $\beta$ defines a character $\chi(\beta)$ on $\Gamma_{v} / 2 \Gamma_{v}$ via

$$
\chi(\beta)\left(v(b)+2 \Gamma_{v}\right)=\operatorname{sign}(\alpha(b)) \operatorname{sign}(\beta(b)),
$$

and $\beta$ extends to $\tilde{\beta}$ as above, if and only if $\chi(\beta)$ can be extended to a character $\chi(\tilde{\beta})$ of $\Gamma_{\tilde{v}} / 2 \Gamma_{\tilde{v}}$. For this it is necessary and sufficient that

$$
T:=\operatorname{Ker}\left(\Gamma_{v} / 2 \Gamma_{v} \rightarrow \Gamma_{\tilde{v}} / 2 \Gamma_{\tilde{v}}\right) \subset \operatorname{Ker}(\chi(\beta)) .
$$

Thus \# $(T)=2^{k}$. Now consider the diagram

$$
\begin{aligned}
& 0 \rightarrow 2 \Gamma_{v} \rightarrow \Gamma_{v} \rightarrow \Gamma_{v} / 2 \Gamma_{v} \rightarrow 0 \\
& \downarrow \alpha \quad \downarrow \beta \quad \downarrow \gamma \\
& 0 \rightarrow 2 \Gamma_{\tilde{v}} \rightarrow \Gamma_{\tilde{v}} \rightarrow \Gamma_{\tilde{v}} / 2 \Gamma_{\tilde{v}} \rightarrow 0
\end{aligned}
$$

Since $\operatorname{Ker}(\beta)=0$ by the snake lemma we get the exact sequence

$$
0 \rightarrow T \rightarrow \operatorname{Coker}(\alpha) \rightarrow \operatorname{Coker}(\beta) \rightarrow \ldots,
$$

but by Proposition $3.1 \mathrm{~b}), \operatorname{Coker}(\beta)=\Gamma_{\tilde{v}} / \Gamma_{v} \simeq I^{\text {dual }}$ is an elementary abelian 2-group. Thus $\operatorname{Coker}(\alpha)$ is mapped to zero. Since $\Gamma_{\tilde{v}}$ is torsion free, $\operatorname{Coker}(\alpha) \simeq \operatorname{Coker}(\beta)$. Concluding, we get $T \simeq I^{\text {dual }}$ which is an elementary abelian 2 -subgroup of $G_{i} / C_{i}$ for some $i \in 1, \ldots, r$.

Case 2. The fan $F$ is not principal.

So there exists $\beta \in F$ which induces $\bar{\beta} \neq \bar{\alpha}$ on $\bar{K}_{v}$. Then $F=F_{1} \cup F_{2}$ is a disjoint union of two fans according to which element on $\bar{K}_{v}$ is induced by $\beta_{i} \in F_{i}$. Also \# $\left(F_{1}\right)=\#\left(F_{2}\right)$. If $F_{1} \cap X \neq \emptyset$ and $F_{2} \cap X \neq \emptyset$ we have $2^{k}=\#\left(F_{i}\right): \#\left(F_{i} \cap X\right)$ for $i=1,2$, hence $2^{k}=\#(F): \#(F \cap X)$ where $2^{k}=\#(I)$.

Now assume, say $F_{2} \cap X=\emptyset$.

Then \# $(F): \#(F \cap X)=2^{k+1}$ where $2^{k}=\#(I)$. So we want to find an elementary abelian extension $H$ of $I$ where $(H: I)=2$. For this note that $\bar{\beta}$ cannot be extended to any $\bar{K}_{\tilde{v}}$ where $\tilde{v}$ is an extension of $v$ to some $L_{i}$. That means $p: \operatorname{Sper}\left(\bar{K}_{\tilde{v}}\right) \rightarrow \operatorname{Sper}\left(\bar{K}_{v}\right)$ is not onto (the extension is not totally real). Now the claim follows from the lemma below (compare Scheiderer [loc. cit.] or [Di-Dr]). In fact, by b) we find a field $\bar{E}$ with $\bar{K}_{v} \supset \bar{E} \supset \bar{K}$ and $\bar{K}_{\tilde{v}} / \bar{E}$ is a non-totally real quadratic extension. Then $\tilde{E}$ is the residue field of a field $E$ with $K^{I} \supset E \supset K^{D}$ and $\left(K^{I}: E\right)=2$. 
Let $H=G(L / E)$. Assume that $\sigma \in H$ has order 4 . Then $L^{\sigma} \not \supset K^{I}$ and thus the residue extensions of $L^{\sigma^{2}} / L^{\sigma}$ and $K^{I} / E$ coincide. But by a) the former is totally real. Contradiction. So $H$ is elementary abelian.

Lemma 3.3. Let $E / F$ be a Galois extension with group $G$.

a) If $G$ is cyclic of order 4 and $E^{\prime}$ is the quadratic subextension of $E / F$, then $E^{\prime} / F$ is totally real.

b) If $E / E^{\sigma}$ is totally real for each involution $\sigma \in G$, then $E / F$ is totally real.

Consider the map $p: \operatorname{Sper}(L) \rightarrow \tilde{X} \subset \operatorname{Sper}(K)$. The group $G$ acts on $\operatorname{Sper}(L)$ and the orbits are just the fibers under $p$. Let $C \subset \operatorname{Sper}(L)$ be constructible and $G$-invariant. Then $p(C)$ is constructible in $\tilde{X}$ and any description of $p(C)$ by elements of $K$ defines automatically a description of $C$. In other words, $C$ can be described by invariant elements. We ask, whether a basic invariant set $C$ can be described by invariant functions as a basic set. One has even more:

Proposition 3.4. Let $C \subset \operatorname{Sper}(L)$ be $G$-invariant and basic, say $C=\left\{a_{1}>0, \ldots\right.$, $\left.\ldots, a_{m}>0\right\}, a_{i} \in L$. Then there are $b_{1}, \ldots, b_{m} \in K$ such that $C=\left\{b_{1}>0, \ldots, b_{m}>0\right\}$.

P r o of. By Proposition 1.4 and the constructibility of $C$ we get by an easy compactness argument that $C$ is basic. We have to describe $p(C) \subset \tilde{X}$ by $k$ inequalities. For this let $F \subset \tilde{X}$ be a finite fan, and let $\tilde{X} \cap p(C) \neq \emptyset$ and $2^{l}=\#(F): \#(\tilde{X} \cap p(C))$. We want $l \leq k$. Let $v$ be the valuation associated to $F, \tilde{v}$ any extension of $v$ to some $L_{i}$. The elements $\alpha \in F$ induce $\bar{\alpha}$ or possibly $\bar{\alpha}$ and $\bar{\beta}$ on $\bar{K}_{v}$. By Proposition 3.1 these can be extended to elements $\alpha^{\prime}$ and $\beta^{\prime} \in \operatorname{Sper}\left(\bar{K}_{\tilde{v}}\right)$ respectively. Now let $F^{\prime}$ be set of all $\tilde{\alpha} \in \operatorname{Sper}\left(L_{i}\right)$ for which one has

$\tilde{\alpha}$ extends some $\alpha \in F$,

$\tilde{\alpha}$ is compatible with $\tilde{v}$,

$\tilde{\alpha}$ induces $\alpha^{\prime}$ (or possibly $\alpha^{\prime}$ or $\beta^{\prime}$ ) on $\bar{K}_{\tilde{v}}$.

Then $F^{\prime}$ is a fan and all fibers of the map $p: F^{\prime} \rightarrow F$ consist of $e=\#(I)$ elements. Since $C$ consists of full fibers and

$$
\#\left(F^{\prime}\right): \#\left(F^{\prime} \cap C\right)=2^{l} \text { with } l \leq k,
$$

we also get

$$
\#(F): \#(F \cap p(C))=2^{l} .
$$

4. Invariant constructible sets. We come back to the situation of Section 1. So $A$ is a commutative ring with unit, $G$ a finite group of automorphisms of $A$ and $B$ is the subring of $G$-invariant elements in $A$. For a finite 2-group $H$ let $d(H)$ be the minimal number of generators. Also, let $k=\sup \{d(H)\}$ where $H$ runs over all 2-subgroups of $G$. This invariant $k=k(G)$ will be important. We assume also that $\operatorname{Sper}(A)$, and thus $\tilde{X} \subset \operatorname{Sper}(B)$, is noetherian. Let $\wp$ be a real prime ideal in $B$ and let $\mathcal{P}_{1}, \ldots, \mathcal{P}_{r}$ be the real extensions of $\wp$ to $A$, that means, the $\mathcal{P}_{i}$ are real prime ideals of $A$ and $\mathcal{P}_{i} \cap B=\wp$ for $i=1, \ldots, r$. Also, let $r>0$. Then $G$ permutes the $\mathcal{P}_{i}$. The residue fields $k\left(\mathcal{P}_{i}\right)$ can be considered as extensions of the residue field $k(\wp)$. Thus $L:=k\left(\mathcal{P}_{1}\right) \oplus \ldots \oplus k\left(\mathcal{P}_{r}\right)$ is a separable $k(\wp)$-algebra on which the group $G$ acts in a natural way. In this situation one has

Proposition 4.1. The subalgebra $L^{G}$ of fixed elements under $G$ coincides with $k(\wp)$. 
Pr o of. Consider the semilocalization

$$
A_{1}:=\left\{\frac{f}{g} \mid f, g \in A, g \notin \mathcal{P}_{1} \cup \ldots \cup \mathcal{P}_{r}\right\} .
$$

Also $G$ acts on $A_{1}$ and $A_{1}^{G}=B_{\wp}$. In fact, for $\frac{f}{g} \in A_{1}^{G}$ we have

$$
\frac{n f}{g}=\frac{\sum_{\sigma \in G} \sigma(f) \prod_{\tau \neq \sigma} \tau(g)}{\prod_{\sigma \in G} \sigma(g)} .
$$

Thus the numerator and denominator are in $A^{G}=B$. Moreover, the denominator is not in $\wp$ since otherwise it would be in $\mathcal{P}_{1}$, hence $\sigma(g) \in \mathcal{P}_{1}$ for some $\sigma \in G$ and $g \in \sigma^{-1}\left(\mathcal{P}_{1}\right)$. Contradiction. Since $\wp$ is real, $n \notin \wp$, thus $f / g \in B_{\wp}$.

So we may replace $A$ by $A_{1}, B$ by $B_{\wp}$ and $\mathcal{P}_{i}$ by $A_{1} \mathcal{P}_{i}, i=1, \ldots, r$. We find elements $l_{i}, i=1, \ldots, r$, with $l_{i} \equiv 1 \bmod \mathcal{P}_{i}$ and $l_{i} \equiv 0 \bmod \mathcal{P}_{j}$ for $j \neq i$. Let $u=\left(a_{1}+\mathcal{P}_{1}, \ldots\right.$, $\left.\ldots, a_{r}+\mathcal{P}_{r}\right) \in \bigoplus_{i=1}^{r} k\left(\mathcal{P}_{i}\right)$ be $G$-invariant. Consider $z=l_{1} a_{1}+\ldots+l_{r} a_{r} \in A$. We have

$$
n u_{i}=(n u)_{i}=\left(\sum_{\sigma \in G} \sigma(u)\right)_{i}=\sum_{\sigma \in G} \sigma(z)+\mathcal{P}_{i} .
$$

Hence $n a_{i}+\mathcal{P}_{i}=a_{i}^{\prime}+\mathcal{P}_{i}$ for $a_{i}^{\prime} \in B$.

Now we can apply the results of the preceding section.

Proposition 4.2. The group $G$ acts transitively on the fibers of the map $p: \operatorname{Sper}(A) \rightarrow \operatorname{Sper}(B)$.

Pr o of. Use Proposition 4.1 and the beginning of Section 3.

Corollary 4.3. Let $C \subset \operatorname{Sper}(A)$ be constructible and $G$-invariant. Then $C$ can be described by $G$-invariant functions.

Pro of. Use Remark 1.3 and take a description of $p(C)$ over $B$.

Next we turn to quantitative questions. Consider the set $\tilde{X}:=p(\operatorname{Sper}(A)) \subset \operatorname{Sper}(B)$. By Remark 1.3, $\tilde{X}$ is constructible and by Proposition 1.2, $\tilde{X}$ is a subspace defined by non-strict inequalities. Hence $\tilde{X}$ is basic closed. By Proposition 4.1 and 3.2, for any real prime ideal $\wp$ of $B$ we get that $\tilde{X} \cap \operatorname{Sper}(k(\wp))$ is generated by $k=k(G)$ elements. If moreover $\operatorname{Sper}(B)$ has finite dimension $d$, we obtain from [A-B-R, V, Theorem 2.9]:

Proposition 4.4. There are $b_{1}, \ldots, b_{m} \in B$ such that $\tilde{X}=p(\operatorname{Sper}(A))=$ $\left\{b_{1} \geq 0, \ldots, b_{m} \geq 0\right\}$ and $m \leq(d+1) k$.

Similarly we get

Proposition 4.5. Let $C \subset \operatorname{Sper}(A)$ be basic closed and $G$-invariant. Assume further that for every real prime ideal $\mathcal{P}$ of $A$ with $\operatorname{dim}(\operatorname{Sper}(A / \mathcal{P}))=i$ the set $\operatorname{Sper}(k(\mathcal{P})) \cap C$ can be written by $s_{i}$ inequalities. Then $C=\left\{b_{1} \geq 0, \ldots, b_{m} \geq 0\right\}$ with $m=\sum_{i=0}^{d} s_{i}$ and $b_{i} \in B$ for $i=1, \ldots, m$.

Pr o of. Again, since $C$ is $G$-invariant, a description of $p(C)$ in $\tilde{X}$ leads to a description of $C$ by $G$-invariant functions. We work in the space of signs $\tilde{X}$ (see [A-B-R, V]). By Propositions 1.1 and $1.3, p(C)$ is basic closed in $\tilde{X}$. The estimate for the number of generators comes from Proposition 3.4 and [A-B-R, V, Theorem 2.9]. 
Remark 4.6. Generically, that means, restricted to real spectra of residue fields at real prime ideals, the estimates in Propositions 4.4 and 4.5 are good. However, the global estimate is unsatisfactory. This is due to the fact that in the case of basic closed sets it is not possible to fit the information on the generic description together in a better way, as one knows from Scheiderer's examples [Schei1], [A-B-R, VI, Examples 7.2]. In the geometric situation, which will be considered in the next section, we will discuss this a little further. Now, for basic open sets, the situation is better:

Theorem 4.7. Let $C \subset \operatorname{Sper}(A)$ be basic open, defined by strict inequalities, and $G$-invariant. Assume further that $p: \operatorname{Sper}(A) \rightarrow \operatorname{Sper}(B)$ is closed. Then $C$ can also be described by strict inequalities with $G$-invariant elements.

Proof. Again, we just need a description for $p(C) \subset \tilde{X}=p(\operatorname{Sper}(A))$. By Proposition 4.1, $p(C)$ is constructible. Let $V \subset \operatorname{Sper}(B)$ be a subvariety (i.e. $V=\operatorname{Var}(\wp):=$ $\{\beta \in \operatorname{Sper}(B) \mid \operatorname{supp}(B) \supset \wp\}$ for a real prime ideal $\wp$ of $B$ with $\left.V \subset \mathrm{Cl}_{Z} \operatorname{Bd}(p(C))\right)$. Note that here we consider the boundary of $p(C)$ in $\tilde{X}$. We want to show that $V \cap p(C)=\emptyset$ in $\tilde{X}$. For this, consider $\beta \in V$ with $\operatorname{supp}(\beta)=\wp$ such that $\beta \in \operatorname{Bd}(p(C))$. There are two cases:

Case $1 . \beta \notin p(C)$. Then there exists $\beta^{\prime} \in p(C)$ which specializes to $\beta: \beta^{\prime} \rightarrow \beta$. Choose $\alpha^{\prime} \in C$ with $p\left(\alpha^{\prime}\right)=\beta^{\prime}$. By going-up (see [A-B-R II, Section 4]) we find a specialization $\alpha$ of $\alpha^{\prime}$ so that $p(\alpha)=\beta$. Thus $\alpha \notin C$, hence $\alpha \in \operatorname{Bd}(C)$. Let $\mathcal{P}:=\operatorname{supp}(\alpha)$ and $W:=\operatorname{Var}(\mathcal{P})$. Then $W \subset \mathrm{Cl}_{Z}(\operatorname{Bd}(C))$ and $p(W)$ is Zariski-dense in $V$. Assume that $\sigma \in V \cap p(C)$. Then we find $\tau \in W \cap C$ with $p(\tau)=\sigma$. But $C$ is basic open, thus $W \cap C=\emptyset$. Contradiction.

Case 2. $\beta \in p(C)$. Now there exists $\beta^{\prime} \in \tilde{X} \backslash p(C)$ which specializes to $\beta$. Choose $\alpha^{\prime} \in \operatorname{Sper}(A) \backslash C$ with $p\left(\alpha^{\prime}\right)=\beta^{\prime}$. Again by going-up we find a specialization $\alpha$ of $\alpha^{\prime}$ so that $p(\alpha)=\beta$. Since $C$ is $G$-invariant, $\alpha \in C$, therefore $\alpha \in \operatorname{Bd}(C)$. But $C$ is open. Contradiction.

So we settled the first assumption of Theorem 2.3. Next, let $F \subset Y$ be a finite fan. We may assume that $\#(F) \geq 4$ for if there are only 2-element fans, then any constructible set $Z \subset Y$ with $Z \cap \mathrm{Cl}_{Z}(\mathrm{Bd}(Z))=\emptyset$ can be described by a single inequality. By Proposition 2.1 all $\sigma \in F$ have the same support, say $\wp$. Now, by Propositions 4.1 and 3.4, $p(C) \cap \operatorname{Sper}(k(\wp))$ can be described by $s$ inequalities. Thus

$$
\#(p(C) \cap F) \text { divides \# }(F)
$$

and

$$
\text { \# }(F) \text { divides } 2^{s} \#(p(C) \cap F) \text {. }
$$

Hence all conditions of Theorem 2.3 are fulfilled and it follows that $p(C)$ can be described by $s$ strict inequalities.

5. Invariant semialgebraic sets over the reals. Let $Y$ be a real algebraic affine $\mathbb{R}$-variety and let $G$ be a compact Lie group which acts on $Y$. We want to study $G$-invariant semialgebraic subsets $C \subset Y$. There is an equivariant algebraic embedding $Y \rightarrow W$ where $W$ is a representation space of $G$ [Kr, II, 2.4]. In other words, we may assume $G \subset 0(n, \mathbb{R})$ and $Y \subset \mathbb{R}^{n}$ is $G$-invariant. By a theorem of Hilbert and Hurwitz [Weyl, VIII, §14] the graded algebra $R[W]^{G}$ of $G$-invariant polynomials is finitely generated, say by homogeneous polynomials $p_{1}, \ldots, p_{m}$. Let $J$ be the ideal of relations of the $p_{i}$ 
in $\mathbb{R}[Y]^{G}$. We set $A:=\mathbb{R}[Y], B:=\mathbb{R}[Y]^{G} \cong \mathbb{R}\left[X_{1}, \ldots, X_{m}\right] / J$ and $Z:=\operatorname{Spec}(B)(\mathbb{R})$. So we get a map $p=\left(p_{1}, \ldots, p_{m}\right): Y \rightarrow Z$. If $C \subset Y$ is semialgebraic, then so is $p(C) \subset Z$ by the Tarski-Seidenberg theorem [B-C-R, §5.2]. Recall that for any semialgebraic set $C \subset Y$ or $C \subset Z$ there corresponds a constructible set $\tilde{C} \subset \operatorname{Sper}(A)$ or $\tilde{C} \subset \operatorname{Sper}(B)$ respectively from which one can read off the properties of $S$ better than from $S$ itself (see $[$ A-B-R, Chap. V, 5]). The canonical map $\operatorname{Sper}(A) \rightarrow \operatorname{Sper}(B)$ will again be denoted by $p$. We set $X:=p(Y)$. Then $X$ is basic closed. More generally, one has (compare [Pro-Schw, Proposition 5.1]):

Proposition 5.1. Let $C \subset Y$ be basic closed and $G$-invariant. Then $p(C)$ is basic closed.

Pr o of. The argument is the same as for Proposition 1.3. For a polynomial $f \in \mathbb{R}[W]$ we set

$$
\operatorname{Tr}(f)(x):=\int_{G} f(g(x)) d g .
$$

Clearly $\operatorname{Tr}(f)$ is $G$-invariant. As before, we get

$$
p(C)^{\sim}=\bigcap_{C \subset\{b \geq 0\}}\{\operatorname{Tr}(b) \geq 0\} .
$$

An easy compactness argument shows that $p(C)^{\sim}$ and thus $p(C)$ is already a finite intersection of sets of the form $\{\operatorname{Tr}(b) \geq 0\}$ with $C \subset\{b \geq 0\}$.

In [Pro-Schw] Procesi and Schwarz give an explicit description of $X=p(Y)$ as follows: The $m \times m$-matrix $P$ with $P_{i j}:=\left\langle\operatorname{grad}\left(p_{i}\right), \operatorname{grad}\left(p_{j}\right)\right\rangle$ has entities in $B=\mathbb{R}[Z]$ (recall that the scalar product $\langle\cdot, \cdot\rangle$ is $G$-invariant by assumption).

Theorem 5.2 (Procesi-Schwarz). $X=\{x \in Z \mid P(x)$ is positive semidefinite $\}$.

We need another important result of Schwarz [Schw]:

Proposition 5.3. The map $p: Y \rightarrow Z$ is proper, it separates orbits of $G$ and induces a homeomorphism $\bar{P}: Y / G \rightarrow X$.

Together with Proposition 5.1 we get

COROLlary 5.4 .

a) Let $C \subset Y$ be semialgebraic and $G$-invariant. Then $C$ can be described by $G$-invariant polynomials.

b) Let $C \subset Y$ be a G-invariant basic (open) closed subset. Then $C$ can be described as an intersection by (strict) non-strict inequalities with $G$-invariant polynomials.

The case of basic open sets requires some explanation. We postpone the proof since we will get a quantitative statement like Theorem 4.7. On the way, we will also find quantitive results for the description of invariant basic closed sets, but these might be not optimal (see Remark 4.6). The result below is a slight generalization of [Pro-Schw, Proposition 7.4] (equivariant Hilbert's 17th problem). 
Corollary 5.5 (equivariant Positivstellensatz). Let $C \subset Y$ be basic closed and $G$-invariant. Then there are finitely many $G$-invariant functions $f_{1}, \ldots, f_{q} \in R[Y]$ such that for any $G$-invariant $f \in \mathbb{R}[Y]$ with $f \geq 0$ on $C$ one has

$$
f^{2 k+1}+f\left(\sum_{i=1}^{q} t_{i} f_{i}\right)-\sum_{i=1}^{q} s_{i} f_{i}=0
$$

for some $k \in \mathbb{N}$ where $t_{i}$ and $s_{i}$ are suitable sums of squares of $G$-invariant elements of $\mathbb{R}[Y]$.

Pro of. Apply the usual Positivstellensatz [B-C-R, 4.4] to $p(C)$.

For a description of $X$ by few inequalities one can use Theorem 5.2. If $G$ is finite, then $d=\operatorname{dim}(Y)=\operatorname{dim}(Z)$. As before, let $m$ be the number of generators for $R[W]^{G}$. Saying that a symmetric $m \times m$ matrix is positive semidefinite requires $m$ non-strict inequalities, since equivalently all coefficients of the characteristic polynomial have to be non-negative.

On the other hand, by Proposition 4.4, generically $\tilde{X}$ and thus $X$ can be described by $k$ inequalities where $k=k(G)$ is defined at the beginning of Section 4. Also, if $Y$ is irreducible and $G$ acts effectively, $k$ can be replaced by the maximal $\mathbb{F}_{2}$-dimension of an elementary abelian 2-group of $G$. So this number $k$ depends only on the structure of $G$ and not on the number $m$ of generators for $R[W]^{G}$. So we got

Proposition 5.6. Let $G$ be finite, acting effectively on the irreducible variety $Y$ and let $k$ be the maximal number for which $G$ contains an elementary abelian subgroup of order $2^{k}$. Then the orbit space $X=p(Y) \subset Z$ is of the form $X=\left\{b_{1} \geq 0, \ldots, b_{k} \geq 0\right\} \cup T$, where $\operatorname{dim}(T)<\operatorname{dim}(Y)$.

Note that $T$ can be chosen to be the orbit spaces of a subfactor of $G$.

For instance, if $\#(G)$ is odd, then $X=Z$ (compare [Pro-Schw, Proposition 3.7]).

Now let us return to the general situation where $G$ is a compact Lie group. We define, however, the invariant $k=k(G)$ again as at the beginning of Section 4. We may assume that $Y \subset \mathbb{R}^{n}$ is compact, for, since $G$ is compact, the action of $G$ on $Y$ can be extended to the one point compactification $\bar{Y}$ of $Y$ (having the point at infinity as a fixed point), and $\bar{Y}$ has the structure of a real affine algebraic variety. We want to describe $X$ by few inequalities. For this we take the corresponding set $\tilde{X}=p(\operatorname{Sper} \mathbb{R}[Y]) \subset \operatorname{Sper} \mathbb{R}[Z]$ and proceed as in Sections 3 and 4: We consider a finite fan $F \in \operatorname{Sper}\left(\mathbb{R}[Y]^{G}\right)$ and compute the index of $(\tilde{X} \cap F)$ in $F$. In fact, as before we get

Lemma 5.7. $\#(F): \#(\tilde{X} \cap F) \leq 2^{k+1}$ where $k=k(G)$.

Proof. If $k=0$, then $G$ is a finite group of odd order, so that $p$ is surjective. Thus the claim is true, if all finite fans are of order $\leq 2$. So let $\#(F) \geq 4$. Then by Proposition 2.1 all $\alpha \in F$ have the same support. Clearly we may assume that $\mathbb{R}[Y]$ and thus $\mathbb{R}[Y]^{G}$ are domains and the support of all $\alpha \in F$ is the zero ideal. (But note that $G$ in general does not act effectively on $Y$. That is the reason why $k(G)$ is not defined just by the order of a maximal elementary abelian 2-group of $G$.) So let us consider $p: \operatorname{Sper}(\mathbb{R}(Y)) \rightarrow \operatorname{Sper}(\mathbb{R}(Z))$. The action of $G$ extends to $\mathbb{R}(Y)$. It can be shown that $\mathbb{R}(Z)$ is the fixed field and the induced action of $G / C$ on $\operatorname{Sper}(\mathbb{R}(Y))$ is free, where $C$ is the centralizer of $\mathbb{R}(Y)$. Correspondingly, we may replace $Y$ and thus $Z$ by monoidal extensions. So by resolution of singularities we may assume that $Y$ is 
smooth. Now assume that $F \cap \tilde{X} \neq \emptyset$ and $F$ is principal. Then there is a closed point $x \in Z$ such that all $\alpha \in F$ are specialized by $x$ (compare [A-B-R, Chap. VI, 6-7]). Since $X$ is closed, we get $x \in X$. After blowing up we may assume that the orbit $p^{-1}(x)$ has maximal dimension, in other words, for $y \in p^{-1}(x)$ the stabilizer $G_{y}$ of $y$ acts as a discrete group on $N_{y}(Y)=T_{y}\left(G_{y}\right)^{\perp} \subset T_{y}(Y)$ where $T_{y}$ is the tangent space in $y$, and orthogonality is with respect to an invariant scalar product in $T_{y}(Y)$. By Luna's slice theorem ([Lu], see also [Slo-Kno]) there is a subvariety $S \subset Y$ having tangent space $T_{y}(S)=N_{y}(Y)$ such that $G_{y}$ acts on $S$ and $p$ defines an étale map $S / / G_{y} \rightarrow Z=Y / / G$. Clearly, $p(S)$ covers a neighborhood of $x$ in $X$. More precisely, let $y^{\prime}=q(y)$ be the image of $y$ under $q: S \rightarrow S / / G_{y}$. Then there is a neighborhood $U$ of $y^{\prime}$ in $S / / G_{y}$ such that $p: U \rightarrow p(U) \subset Z$ is a Nash-isomorphism. Since all $\alpha \in F$ are specialized by $x$ we have $F \cap \tilde{X}=F \cap p(S)^{\sim}$

Claim. The fan $F$ lifts uniquely to a fan $F^{\prime}$ in $\operatorname{Sper}\left(\mathbb{R}\left(S / / G_{y}\right)\right)$ such that all $\beta^{\prime} \in F^{\prime}$ are centered at $y^{\prime}$.

In order to see this take an element $\alpha \in F \cap X$ and an extension $\alpha^{\prime}$ of $\alpha, \alpha^{\prime} \in$ $\operatorname{Sper}\left(\mathbb{R}\left(S / / G_{y}\right)\right)$ such that $\alpha^{\prime}$ is centered at $y^{\prime}$. Let $v$ be the valuation associated to $F$ and $v^{\prime}$ the extension of $v$ to $\mathbb{R}\left(S / / G_{y}\right)$ which is compatible with $\alpha^{\prime}$. By assumption $v^{\prime}$ is unramified over $v$. Therefore the kernel of $\Gamma_{v} / 2 \Gamma_{v} \rightarrow \Gamma_{v^{\prime}} / 2 \Gamma_{v^{\prime}}$ is automatically contained in the kernel of any character of $\Gamma_{v} / 2 \Gamma_{v}$. By the Baer-Krull theorem (see Section 3) it follows that all $\beta \in F$ can be extended to $\beta^{\prime}$ in such a way that $\beta^{\prime}$ is compatible with $v^{\prime}$ and induces the same ordering than $\alpha^{\prime}$ on $\bar{K}_{v^{\prime}}$. This settles the claim.

Now by Proposition 4.4 we have

$$
\#(F): \#\left(F \cap p(S)^{\sim}\right)=\#\left(F^{\prime}\right): \#\left(F^{\prime} \cap q(S)^{\sim}\right) \leq 2^{k\left(G_{y}\right)} .
$$

But $k\left(G_{y}\right) \leq k(G)$. This settles the claim for principal fans. Since a non-principal fan $F$ splits into two principal fans of the same size, the worst thing that can happen is that $\#(F): \#\left(F \cap p(S)^{\sim}\right)=2^{k(G)+1}$.

Unfortunately, I was not able to handle the non-principal fans as in the proof of Proposition 4.4. In the same way as Proposition 5.5 we get

Proposition 5.8. Let $G$ be a compact Lie group, acting effectively on the irreducible variety $Y$ and let $k$ be the maximal number for which $G$ contains an elementary abelian subgroup of order $2^{k}$. Then the orbit space $X=p(Y) \subset Z$ has the form $X=\left\{b_{1} \geq 0, \ldots\right.$, $\left.\ldots, b_{k+1} \geq 0\right\} \cup T$ with $b_{i} \in \mathbb{R}[Z]$ and $\operatorname{dim}(T)<\operatorname{dim}(Z)$.

In fact, in all examples I know one needs only $k$ inequalities and no $T$. Of course, if $Y$ is smooth, or if the regular points are dense in $Y$ one can write $X$ as the closure of a basic open set of the form $\left\{b_{1}>0, \ldots, b_{k+1}>0\right\}$. Let us continue with the situation in the proof of Lemma 5.7. A fan $F \subset \tilde{X} \cap \operatorname{Sper}(\mathbb{R}(Z))$ lifts uniquely to a fan $F^{\prime}$ in $\operatorname{Sper}\left(\mathbb{R}\left(S / / G_{y}\right)\right)$ such that all $\beta^{\prime} \in F^{\prime}$ are centered at $y^{\prime}$. This is as in the claim but $F$ is not the same fan. Here we consider only fans which are completely contained in $\tilde{X}$. Now $F^{\prime}$ lifts to a fan $\tilde{F} \subset \operatorname{Sper}(\mathbb{R}(S))$ such that all $\tilde{\beta} \in \tilde{F}$ are centered at $y$. Moreover, the fibers of the map $p: \tilde{F} \rightarrow F$ consist of $e$ elements where $e$ is the ramification index of $v^{\prime}$ for the Galois extension $\mathbb{R}(S) / \mathbb{R}\left(S / / G_{y}\right)$ and $v^{\prime}$ is the valuation associated to $F^{\prime}$ (compare the proof of Proposition 3.4). 
Now let $C \subset Y$ be basic (open or closed), described by $s$ elements and let $C$ be $G$-invariant. Then

$$
\tilde{C} \cap \tilde{F}=\emptyset \text { or } \#(\tilde{F}):(\tilde{C} \cap \tilde{F})=2^{t} \text { with } t \leq s .
$$

Hence $p(C)^{\sim} \cap F=\emptyset$ or $\#(F): \#\left(F \cap p(C)^{\sim}\right)=2^{t}$. Next consider a fan $F \subset \tilde{X} \cap$ $\operatorname{Sper}(\mathbb{R}(Z))$ which is not centered at one point. So $F$ is not principal. As before we get

$$
F \cap p(C)^{\sim}=\emptyset \text { or } \#(F): \#(F \cap p(S))^{\sim}=2^{t} \text { with } t \leq s+1 .
$$

Using again [A-B-R, V, Theorem 2.9] we obtain

Proposition 5.9. Let $C \subset Y$ be basic closed, say $C=\left\{a_{1} \geq 0, \ldots, a_{s} \geq 0\right\}$ with $a_{i} \in \mathbb{R}[Y]$ and assume that $C$ is $G$-invariant. Then $C$ can be written in the form $C=$ $\left\{b_{1} \geq 0, \ldots, b_{l} \geq 0\right\}$ with $b_{i} \in \mathbb{R}[Y]^{G}$ and $l \leq(s+1)\left(d-\frac{1}{2} s\right)$ for $s<d, l \leq \frac{1}{2} d(d+1)$ for $s \geq d$, where $d=\operatorname{dim}(Z)$.

Again I do not know any example where one cannot choose $l=s$. Also, up to a set of smaller dimension, $C$ can be described by $s+1$ invariant inequalities.

Now let $C \subset Y$ be basic open. Since $p$ is proper, as in the proof of Theorem 4.7 we see that $p(C) \cap \mathrm{Cl}_{Z}(\operatorname{Bd}(p(C))=\emptyset$ where again the boundary has to be taken in the interior of $X$. Hence we get from Lemma 5.7 and Theorem 2.3:

Theorem 5.10. Let $C \subset Y$ be G-invariant and basic open, say $C=\left\{a_{1}>0, \ldots\right.$, $\left.\ldots, a_{s}>0\right\}$ with $a_{i} \in \mathbb{R}[Y]$. Then $C$ can also be written in the form $C=\left\{b_{1}>0, \ldots\right.$, $\left.\ldots, b_{s+1}>0\right\}$ with $b_{i} \in \mathbb{R}[Z]=\mathbb{R}[Y]^{G}$.

\section{References}

[A-B-R] C. Andradas, L. Bröcker, J. Ruiz, Constructible Sets in Real Geometry, Ergeb. Math. Grenzgeb. (3) 33, Springer, Berlin, 1996.

[Be1] E. Becker, Hereditarily-Pythagorean Fields and Orderings of Higher Level, Monografias de Matemática 29, Instituto de Matématica Pura et Aplicada, Rio de Janeiro, 1978.

[Be2] E. Becker, On the real spectrum of a ring and its applications to semi-algebraic geometry, Bull. Amer. Math. Soc. (N.S.) 15 (1986), 19-60.

[B-C-R] J. Bochnak, M. Coste, M.-F. Roy, Géométrie algébrique réelle, Ergeb. Math. Grenzgeb. (3) 12, Springer, Berlin, 1987.

[B] L. Bröcker, On basic semialgebraic sets, Exposition. Math. 9 (1991), 289-334.

[DM-I] F. De Meyer, E. Ingraham, Separable Algebras over Commutative Rings, Lecture Notes in Math. 181, Springer, Berlin, 1971.

[Di-Dr] J. Diller, A. Dress, Zur Galoistheorie pythagoreischer Körper, Arch. Math. (Basel) 16 (1965), 148-152.

[Kn-Schei] M. Knebusch, C. Scheiderer, Einführung in die reelle Algebra, Vieweg, Braunschweig, 1989.

[Kr] H. Kraft, Geometrische Methoden in der Invariantentheorie, Aspects of Math. D1, Vieweg, Braunschweig, 1985.

[Lu] D. Luna, Slices étales, in: Sur les groupes algébriques, Bull. Soc. Math. France, Mémoire 33, Paris, 1973, 81-105. 
[Mar] M. Marshall, Minimal generation of basic sets in the real spectrum of a commutative ring, in: Recent Advances in Real Algebraic Geometry and Quadratic Forms, Contemp. Math. 155, Amer. Math. Soc., Providence, 1994, 207-219.

[Pr] A. Prestel, Lectures on Formally Real Fields, Lecture Notes in Math. 1093, Springer, Berlin, 1984.

[Pro-Schw] C. Procesi, G. Schwarz, Inequalities defining orbit spaces, Invent. Math. 81 (1985), $539-554$.

[Schei1] C. Scheiderer, Stability index of real varieties, Invent. Math. 97 (1989), 467-483.

[Schei2] C. Scheiderer, Spaces of orderings of fields under finite extensions, Manuscripta Math. 72 (1991), 27-47.

[Schw] G. Schwarz, The topology of algebraic quotients, in: Topological Methods in Algebraic Transformation Groups, Progr. Math. 80, Birkhäuser, Boston, 1989, 135-152.

[Slo-Kno] P. Slodowy, Der Scheibensatz für algebraische Transformationsgruppen, mit einem Anhang von F. Knop, in: Algebraische Transformationsgruppen und Invariantentheorie, DMV Sem. 13, Birkhäuser, Basel, 1989, 89-114.

[Weyl] H. Weyl, The Classical Groups, 2nd ed., Princeton University Press, 1946. 